\title{
T helper cells implicated in early Huntington disease
}

The presymptomatic stage of Huntington disease (HD) is marked by an increased prevalence of T helper 17.1 ( $\left.\mathrm{T}_{\mathrm{H}} 17.1\right)$ cells in the cerebrospinal fluid (CSF), according to a new study published in Annals of Neurology. The finding suggests that therapies that reduce $\mathrm{T}$ cell-driven inflammation could delay or prevent onset of the disease.

$\mathrm{HD}$ is an inherited neurodegenerative disease caused by an expanded trinucleotide repeat in the HTT gene, which encodes the huntingtin protein. The pathogenesis of HD is still unclear, but evidence of a role for immune abnormalities is accumulating.

"Studies have demonstrated the expression of mutated huntingtin in cells of the immune system, where the protein has pro-inflammatory properties," explains Finn Sellebjerg, who led the new study alongside Marina von Essen. “This observation has led several research groups to investigate the possibility of immune activation within the CNS in $\mathrm{HD}$, and whether this activation is involved in the pathogenesis of the disease."

The researchers analysed CSF from individuals carrying the HTT expansion, some of whom had not yet developed symptoms of the disease, and from healthy noncarriers. In HTT expansion carriers, levels of the cytokines LTa and IL-17 were higher than in the healthy controls, indicating greater proinflammatory lymphocyte activity in these individuals.

The frequency of proinflammatory $\mathrm{T}_{\mathrm{H}} 17.1$ cells in the CSF was higher in individuals with the HTT expansion than in healthy controls. Carriers of the HTT expansion who had not yet developed the motor symptoms of HD had more CSF $\mathrm{T}_{\mathrm{H}} 17.1$ cells than those with motor symptoms, indicating

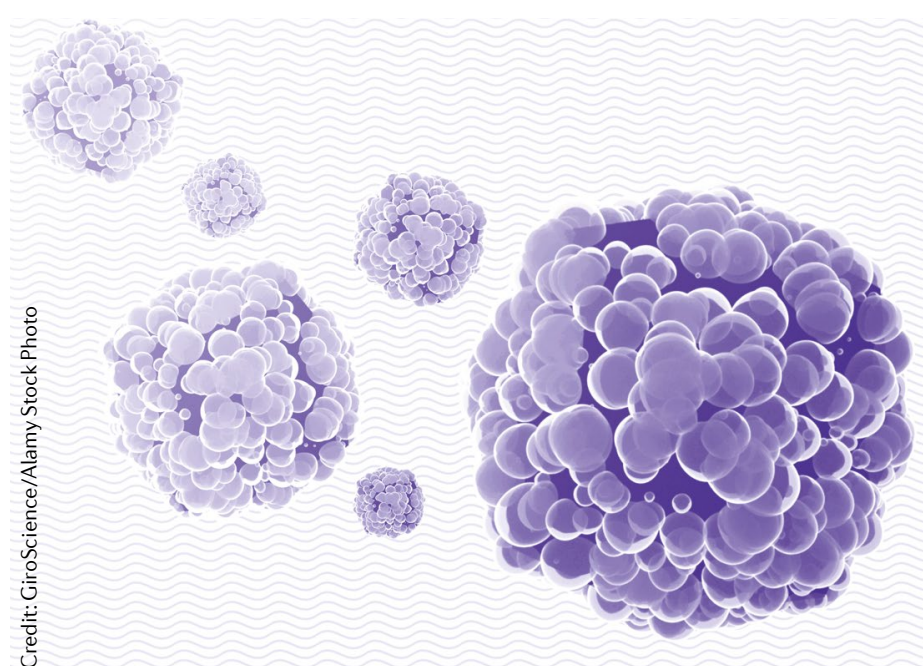

that these cells are involved in the very early stages of the disease.

"The study raises the question of whether immunomodulatory or immunosuppressive therapy

Studies have demonstrated the expression of mutated huntingtin in cells of the immune system 5 before the onset of HD may delay or even prevent the onset of disease in carriers of the pathogenic gene expansion," concludes von Essen.

Sarah Lemprière

\section{NLRP3 inflammasome activation implicated in tau pathology}

Activation of the NLRP3 inflammasome in microglia can drive tau pathology in a mouse model of frontotemporal dementia (FTD), according to a new study published in Nature. The findings also shed light on the role of microglia in Alzheimer disease (AD).

The NLRP3 inflammasome is a multiprotein complex consisting of NLRP3, ASC and caspase 1 . The complex is assembled in activated microglia and induces release of the proinflammatory cytokine IL-1 $\beta$. Evidence suggests that NLRP3 inflammasome activation is involved in the development of amyloid- $\beta(A \beta)$ pathology in $A D$, but whether activity of this inflammasome also affects tau pathology is not clear. The new study aimed to address this uncertainty.

"Innate immune reactions occur in most neurodegenerative diseases,
Innate immune reactions occur in most neurodegenerative diseases

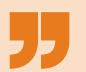

ORIGINAL ARTICLE von Essen, M. R. et al. Early intrathecal Thelper 17.1 cell activity in Huntington's disease. Ann. Neurol. https://doi.org/ 10.1002/ana.25647 (2019) so we were interested in whether the NLRP3 signalling would be

NLRP3 activation in the brain was higher in Tau22 mice than in wild-type mice. Furthermore, 'inflammasome knockout' Tau22 mice, which were deficient in either ASC or NLRP3, had lower levels of tau pathology and did not show spatial memory deficits.

Finally, hippocampal injection of $\mathrm{A} \beta$-containing brain homogenate induced tau pathology in Tau22 mice, but not in inflammasome-knockout Tau22 mice. This observation agrees with existing evidence that $A \beta$ pathology induces tau pathology in AD and implicates the NLRP3 inflammasome in this process.

"These data identify an important role of microglia and NLRP3 inflammasome activation in the pathogenesis of tauopathies and support the amyloid-cascade hypothesis in $A D$, demonstrating that neurofibrillary tangles develop downstream of $A \beta$-induced microglial activation," say the researchers in the paper.

Sarah Lemprière activation in the Tau22 mouse model of FTD. These mice overexpress mutated forms of human tau, and develop tau pathology and spatial memory deficits.
ORIGINAL ARTICLE Ising, C. et al. NLRP3

inflammasome activation drives tau pathology. Nature 575, 669-673 (2019) 